\title{
The role of surgical cytoreduction in the treatment of malignant pleural mesothelioma: Meeting summary of the International Mesothelioma Interest Group Congress, September 11- 14, 2012, Boston, Mass
}

\author{
Valerie Rusch, MD, ${ }^{\mathrm{a}}$ Elizabeth H. Baldini, MD, MPH, ${ }^{\mathrm{b}}$ Raphael Bueno, MD,${ }^{\mathrm{c}}$ Marc De Perrot, MD, ${ }^{\mathrm{d}}$ \\ Raja Flores, MD, ${ }^{\mathrm{e}}$ Seiki Hasegawa, MD ${ }^{\mathrm{f}}$ Walter Klepetko, MD, ${ }^{\mathrm{g}}$ Lee Krug, MD, ${ }^{\mathrm{h}}$ Loïc Lang-Lazdunski, MD, \\ PhD, FRCS (Eng), ${ }^{\mathrm{i}}$ Harvey Pass, MD,${ }^{\mathrm{j}}$ Walter Weder, MD,${ }^{\mathrm{k}}$ and David J. Sugarbaker, MD, ${ }^{\mathrm{c}}$ on behalf of the \\ participants in the 2012 International Mesothelioma Interest Group Congress
}

The treatment of all solid tumors, including malignant pleural mesothelioma (MPM), is dependent on (1) macroscopic complete resection and (2) treatment of micrometastatic disease. The role of surgery in the treatment of MPM has been the subject of debate after the recent publication of the Mesothelioma and Radical Surgery (MARS) I trial. ${ }^{1}$ The International Mesothelioma Interest Group (IMIG) met from September 11 through 14, 2012, in Boston, Mass. During this meeting, more than 500 participants representing all the involved specialty groups met in multiple comprehensive sessions to review, critique, and extend the state of knowledge regarding the role of surgery, including both extended pleurectomy/decortication (P/D) and extrapleural pneumonectomy (EPP), in the treatment of MPM.

Some of the deficiencies of the MARS I trial, which was published a year ago in Lancet Oncology, were discussed in multiple sessions of the IMIG meeting. The editorial that accompanied the publication articulated numerous shortcomings of the trial. ${ }^{2}$ The MARS I trial was designed as a pilot feasibility trial, the result of which was negative in that it failed to demonstrate the feasibility of randomly allocating patients to surgery versus no surgery. Nevertheless, the

\footnotetext{
From the Thoracic Service, ${ }^{\mathrm{a}}$ Department of Surgery, Memorial Sloan-Kettering Cancer Center, New York, NY; Department of Radiation Oncology, ${ }^{\mathrm{b}}$ Dana Farber Cancer Institute, Boston, Mass; Division of Thoracic Surgery, ${ }^{\mathrm{c}}$ Brigham and Women's Hospital, Boston, Mass; Division of Thoracic Surgery, ${ }^{\mathrm{d}}$ Toronto General Hospital and Princess Margaret Hospital, University Health Network, Toronto, Ontario, Canada; Division of Thoracic Surgery, ${ }^{\mathrm{e}}$ Mount Sinai Medical Center, New York, NY; Department of Surgery, ${ }^{\mathrm{f}}$ Hyogo College of Medicine, Nishinomiya Hyogo, Japan; Division of Thoracic Surgery, ${ }^{\mathrm{g}}$ Department of Surgery, Comprehensive Cancer Center, Medical University of Vienna,Vienna, Austria; Department of Medicine, ${ }^{\text {h }}$ Memorial Sloan-Kettering Cancer Center and Weill Cornell Medical College, New York, NY; Department of Thoracic Surgery, ${ }^{i}$ Guy's Hospital, London, United Kingdom; Department of Cardiothoracic Surgery, ${ }^{j}$ New York University, Langone Medical Center, New York, NY; and Division of Thoracic Surgery, ${ }^{k}$ University Hospital Zurich, Zurich, Switzerland.

Disclosures: Authors have nothing to disclose with regard to commercial support.

Address for reprints: David J. Sugarbaker, MD, Division of Thoracic Surgery, Brigham and Women's Hospital, 75 Francis St, Boston, MA 02115 (E-mail: dsugarbaker@partners.org).

J Thorac Cardiovasc Surg 2013;145:909-10

$0022-5223 / \$ 36.00$

Copyright (C) 2013 by The American Association for Thoracic Surgery

http://dx.doi.org/10.1016/j.jtcvs.2013.01.039
}

publication contained an analysis of tertiary end points, including survival, which was based on the small pilot cohort, representing fewer than $10 \%$ of the required sample size for an adequately powered between-arm comparison as published by the MARS trialists. Protocol compliance was also poor in that 6 of 26 patients in the no EPP group underwent off-protocol surgery, whereas only 16 of 24 patients in the EPP group actually underwent EPP.

Quality control of the surgery in the MARS trial, if undertaken, was not reported. Intent-to-treat morbidity (11/24; $46 \%)$ and mortality $(3 / 24 ; 13 \%)$, and more strikingly, EPP-associated morbidity $(11 / 16 ; 69 \%)$ and mortality $(3 / 16 ; 19 \%)$, were much higher than reported in the literature. The chemotherapy regimens applied were uncontrolled. Neither final histologic type nor disease stage was reported for the patients who underwent surgery, leaving an open question as to whether these patients, who demonstrated survival inferior to most previous reports, may have had disproportionate N2 or nonepithelial disease. Conversely, the reported 19-month median survival among chemotherapyonly (no EPP) patients was clearly anomalous when compared with a vast prospective literature. The long-term outcome of the study cohort remains unknown, because the overall survival analysis was truncated at 18 months, whereas the quality of life data were reported to 24 months. These deficiencies make drawing any conclusions from MARS I regarding the therapeutic efficacy of EPP impossible.

The patterns of failure in MPM were reaffirmed at the 2012 IMIG meeting. Dr Elizabeth H. Baldini, in reference to her previous work, presented a contemporary group of patients and demonstrated essentially the same distribution of recurrence as originally reported, which is primarily lo$\mathrm{cal}^{3}{ }^{3} \mathrm{Six}$ institutional series from the US, Europe, and Japan involving macroscopic complete resection by EPP or P/D in the setting of multimodality treatment of MPM were presented at the meeting. ${ }^{4-9}$ These reports were discussed in detail in light of previous literature to date. Median survival ranged from 25 to 37 months for patients with epithelial disease and negative extrapleural lymph nodes. Operative mortality ranged from $0 \%$ to $2 \%$. 
On behalf of the International Association for the Study of Lung Cancer (IASLC), Dr Valerie Rusch presented a preliminary analysis of the IASLC staging project, which has since been published in the November 2012 issue of the Journal of Thoracic Oncology. ${ }^{10}$ In the IASLC worldwide registry of patients with all stages of epithelial MPM, the analysis showed 19-month median survival among 1359 patients undergoing surgical resection (P/D or EPP). Moreover, patients undergoing EPP for early-stage disease demonstrated survival superior to that of all other subgroups, a median of 40 months. On the basis of the current literature and the IASLC report, it was concluded by IMIG members that surgery, whether P/D or EPP, with the goal of obtaining a macroscopic complete resection should be performed in the multimodality treatment of MPM. In particular, it was agreed that the type of cytoreductive procedure should be selected on the basis of disease distribution, institutional experience, and surgeon preference and experience. Furthermore, it was collectively decided that these operations should be performed by surgeons who have achieved morbidity and mortality within the scope of the current literature.

After much discussion in multiple forums and settings with surgeons, medical oncologists, radiation oncologists, epidemiologists, and basic scientists, the attendees of the 2012 IMIG meeting reached agreement on the following points:

- Surgical macroscopic complete resection and control of micrometastatic disease play a vital role in the multimodality therapy of MPM, as is the case for other solid malignancies.

- Surgical cytoreduction is indicated when macroscopic complete resection is deemed achievable.

- The type of surgery (EPP or P/D) depends on clinical factors and on individual surgical judgment and expertise.

- All patients with the diagnosis of MPM should be initially evaluated in a multidisciplinary setting, including medical oncology, radiation oncology, and surgery.

- Clinical staging (lymph node sampling, positron emission tomography, magnetic resonance imaging) should be performed before therapy.

- The histologic subtype should be identified by tissue biopsy before initiation of therapy.

\section{References}

1. Treasure T, Lang-Lazdunski L, Waller D, Bliss JM, Tan C, Entwisle J, et al. Extra-pleural pneumonectomy versus no extra-pleural pneumonectomy for patients with malignant pleural mesothelioma: clinical outcomes of the Mesothelioma and Radical Surgery (MARS) randomised feasibility study. Lancet Oncol. 2011;12:763-72.

2. Weder W, Stahel RA, Baas P, Dafni U, de Perrot M, McCaughan BC, et al. The MARS feasibility trial: conclusions not supported by data. Lancet Oncol. 2011; 12:1093-4; author reply 1094-5.

3. Goodman BM, Gill RR, Winfrey O, Richards WG, Chen AB, Kozono DE, et al. Patterns of recurrence following extrapleural pneumonectomy (EPP) for malignant pleural mesothelioma (MPM). In: 11th International Conference of the International Mesothelioma Interest Group abstract book. Boston: iMig; 2012. Available at: http://www.imig2012.org/documents/ iMig2012_AbstractBook_vF_Sept10_REV.pdf. Abstract IIIA.2.

4. Bölükbas S, Eberlein M, Kudelin N, Fisseler-Eckhoff A, Schirren J. Long-term outcome after radical pleurectomy followed by chemoradiation for malignant pleural mesothelioma: a 10-year single center experience. In: 11th International Conference of the International Mesothelioma Interest Group abstract book. Boston: iMig; 2012. Available at: http://www.imig2012.org/documents/ iMig2012_AbstractBook_vF_Sept10_REV.pdf. Abstract IIB.2.

5. Sugarbaker DJ, Dasilva M, Supko J, Winfrey O, Eisen H, Barlow J, et al. Prospective phase I trial of extrapleural pneumonectomy or pleurectomy/decortication, intrathoracic/intraperitoneal hyperthermic [HIOC] cisplatin and gemcitabine with intravenous amifostine and sodium thiosulfate cytoprotection for patients with resectable malignant pleural mesothelioma. In: 11th International Conference of the International Mesothelioma Interest Group abstract book. Boston: iMig; 2012. Available at: http://www.imig2012.org/documents/ iMig2012_AbstractBook_vF_Sept10_REV.pdf. Abstract IIB.4.

6. Friedberg J, Culligan MJ, Mick R, Hahn SM, Stevenson J, Alley E, et al. Radical pleurectomy and intraoperative porfimer sodium photodynamic therapy for malignant pleural mesothelioma. In: 11th International Conference of the International Mesothelioma Interest Group abstract book. Boston: iMig; 2012. Available at: http://www.imig2012.org/documents/iMig2012_AbstractBook_ vF_Sept10_REV.pdf. Abstract IIIA.4.

7. Cale A, Qadri S, Cowen M, Wieczorek A, Lind MJ, et al. Is there life after MARS? Does extrapleural pneumonectomy still have a role in the management of malignant pleural mesothelioma-a 13 years single centre experience. In: 11th International Conference of the International Mesothelioma Interest Group abstract book. Boston: iMig; 2012. Available at: http://www.imig2012.org/documents/ iMig2012_AbstractBook_vF_Sept10_REV.pdf. Abstract P1.01.

8. Hasegawa S, Tanaka F, Kondo N, Okumura Y, Matsumoto S, Takuwa T, et al. Outcome of consecutive 61 cases of intent-to-treat with extrapleural pneumonectomy for resectable malignant pleural mesothelioma. In: 11th International Conference of the International Mesothelioma Interest Group abstract book. Boston: iMig; 2012. Available at: http://www.imig2012.org/documents/iMig2012_ AbstractBook_vF_Sept10_REV.pdf. Abstract P1.05.

9. Bille' A, Okiror L, Spicer J, Steele JP, Landau D, Taylor H, et al. Pleurectomy/ decortication, hyperthermic pleural lavage with povidone-iodine followed by adjuvant chemotherapy: survival analysis in 65 consecutive patients. In: 11 th International Conference of the International Mesothelioma Interest Group abstract book. Boston: iMig; 2012. Available at: http://www.imig2012.org/documents/ iMig2012_AbstractBook_vF_Sept10_REV.pdf. Abstract P3.18.

10. Rusch VW, Giroux D, Kennedy C, et al. Initial analysis of the International Association for the Study of Lung Cancer mesothelioma database. J Thorac Oncol. 2012;7:1631-9. 